\title{
Classification of ELM types in Joint European Torus based on global plasma parameters using discriminant analysis
}

\author{
Aqsa Shabbir ${ }^{\mathrm{a}, \mathrm{b}}$, Gregoire Hornung ${ }^{\mathrm{a}}$, Jean-Marie Noterdaeme ${ }^{\mathrm{a}, \mathrm{b}}$, Geert Verdoolaege ${ }^{\mathrm{a}, \mathrm{c}, *}$, JET contributors ${ }^{1}$ \\ EUROfusion Consortium, JET, Culham Science Centre, Abingdon, OX14 3DB, UK \\ ${ }^{a}$ Department of Applied Physics, Ghent University, B-9000 Ghent, Belgium \\ ${ }^{b}$ Max Planck Institute for Plasma Physics, D-85748 Garching, Germany \\ ${ }^{c}$ Laboratory for Plasma Physics, Royal Military Academy, B-1000 Brussels, Belgium
}

\begin{abstract}
In this work, discriminant analysis is used as the main approach for building a physics based automated classifier for the discrimination of the edge-localized mode (ELM) plasma instability. The classifier is then applied for distinguishing type I and type III ELMs from a set of carbon-wall plasmas at JET. This provides a fast, standardized classification of ELM types which is expected to significantly reduce the effort of ELM experts in identifying ELM types. Further, the classifier yields a separation hyperplane in terms of global plasma parameters, which provides an insight into the range of conditions under which specific ELM behaviors occur.
\end{abstract}

Keywords: Edge-localized modes, discriminant analysis, classification, JET

\section{Introduction}

Edge-localized modes (ELMs) are magnetohydrodynamic instabilities occurring in the edge region of high confinement fusion plasmas. ELMs lead to the ejection 5 of energy and particles from the plasma core and onto the plasma-facing components (PFCs). While they are 30 beneficial for impurity regulation, in future devices, such as ITER, large unmitigated ELMs will lead to intolerable heat loads on the PFCs.

10 A first characterization of ELMs is the identification of their type. In this work, a statistical method, discrimi- 35 nant analysis (DA) is employed for developing a simple predictive algorithm for distinguishing ELM types. As an application of our analysis, we discriminate between type I and type III ELMs in a set of carbon-wall (CW) plasmas from the Joint European Torus (JET) tokamak. ${ }^{40}$ Previously, several efforts have been made to statistically characterize [1] 2] and provide an automated classification scheme for ELMs [3] 4] [5]. Herein, the advantage with respect to earlier ELM classification works is twofold. First, we rely on routinely measured global plasma parameters. ${ }^{45}$ Secondly, compared to other classifiers that may be more accurate, DA yields a separation hyperplane between ELM types, we obtain an analytical expression enabling verifi-

\footnotetext{
* Corresponding author

Email address: geert.verdoolaege@ugent.be (Geert Verdoolaege )

${ }^{1}$ See the Appendix of F. Romanelli et al., Proceedings of the 25 th IAEA Fusion Energy Conference 2014, Saint Petersburg, Russia.
}

parameters, as well as quantification of the significance of each parameter. Our approach is intended as a tool to support planning and analysis of experiments.

\section{Discriminant analysis}

In this section, background on the application of discriminant analysis (DA) 6 to the discrimination of ELM types is given. DA enables prediction of the class membership (ELM type) based on a linear or a quadratic combination of plasma parameters. DA is a parametric method and assumes that the distribution of the plasma parameters within each class is multivariate normal.

In this work, two classes $(k=1,2)$ of plasmas with type I and type III ELMs are considered. Each class $k$ with $n_{k}$ plasmas is denoted by a $n_{k} \times p$ data matrix, where $p$ is the number of plasma parameters. The class-specific probability density of a plasma $\mathbf{x}$ belonging to class $k=r$ is denoted as $f_{r}(x)$. Further, $\pi_{r}$ denotes the prior probability of plasma $\mathbf{x}$ belonging to class $r$, with $\Sigma_{r=1}^{k} \pi_{r}=1$. The posterior probability of a plasma $\mathbf{x}$ belonging to class $k=r$ is obtained by applying Bayes' theorem:

$$
P(r \mid x)=\frac{f_{r}(x) \pi_{r}}{\sum_{s=1}^{k} f_{s}(x) \pi_{s}} .
$$

The denominator is consistent across all classes; hence it suffices to estimate class-specific densities $f_{r}(x)$ for each of the classes. It follows that we classify $\mathbf{x}$ in class $r$ if $f_{r}(x) \pi_{r}$ is maximal. Each of the class densities is modeled as a multivariate normal density:

$$
f_{r}(x)=\frac{1}{(2 \pi)^{p / 2} \sqrt{\left|\Sigma_{r}\right|}} \exp \left(-\frac{1}{2} d_{r}^{2}(x)\right) .
$$


The Mahalanobis distance of a plasma $\mathbf{x}$ to class $r$ is given as

$$
d_{r}(x)=\sqrt{\left(x-\mu_{r}\right)^{t}\left(\Sigma_{r}\right)^{-1}\left(x-\mu_{r}\right)} .
$$

Linear discriminant analysis (LDA). All classes are considered to be sharing a common covariance matrix. Hence, $\Sigma_{r}=\Sigma$ for all classes $r$. Taking the logarithm of $f_{r}(x) \pi_{r}$ and after simplifying we obtain for each class, the class scores $l_{r}(x)$ given by

$$
l_{r}(x)=x^{t} \Sigma^{-1} \mu_{r}-\frac{1}{2}\left(\mu_{r}\right)^{t} \Sigma^{-1} \mu_{r}+\log \left(\pi_{r}\right) .
$$

The score $l_{r}(x)$ is a linear function of $x$ and the decision boundary between two classes is the collection of points $\mathbf{x}$ for which $l_{r}(x)=l_{s}(x)$. In $p$ dimensions the boundary between two classes is thus a hyperplane. The class centers and the common covariance matrix for the classes are estimated from the plasmas for which the ELM type is known (training data). The standard estimates are:

$$
\begin{aligned}
& \mu_{r}=\frac{1}{n_{r}} \sum_{k_{i}=r} x_{i} \\
& \Sigma=\frac{1}{n-k} \sum_{r=1}^{k} \sum_{k_{i}=r}\left(x_{i}-\mu_{r}\right)\left(x_{i}-\mu_{r}\right)^{t} .
\end{aligned}
$$

Prior class probabilities are also estimated from the data and are given as:

$$
\pi_{r}=\frac{n_{r}}{n}
$$

where $n_{r}$ is the number of plasmas belonging to class $r^{105}$ and $n$ is the total number of plasmas in the data.

Quadratic discriminant analysis (QDA). Classes do not share a common covariance matrix and the class score ${ }^{110}$ $q_{r}(x)$ is a quadratic function of $\mathbf{x}$. Hence, the decision

so boundary between any two classes $q_{r}(x)=q_{s}(x)$ is also quadratic. Again, the covariance matrix for each class is estimated by the sample covariance matrix of the training samples in that class.

\section{Classification of ELM types}

Dataset. A dataset comprising 74 type I and 26 type III ELMy plasmas spanning over the shot range [50564-76483] $]_{120}$ was assembled from the JET CW experiments. This is an extension of the data set used earlier by Webster et al. [1] and is the same dataset that was used earlier for the visualization of the tokamak operational space in 77. The analysis, in this work, has been restricted to time intervals in which the plasma conditions are quasistationary with approximately constant heating, gas fueling and cen-125 tral density. Further, all experiments dealing with ELM control and mitigation techniques have been excluded.
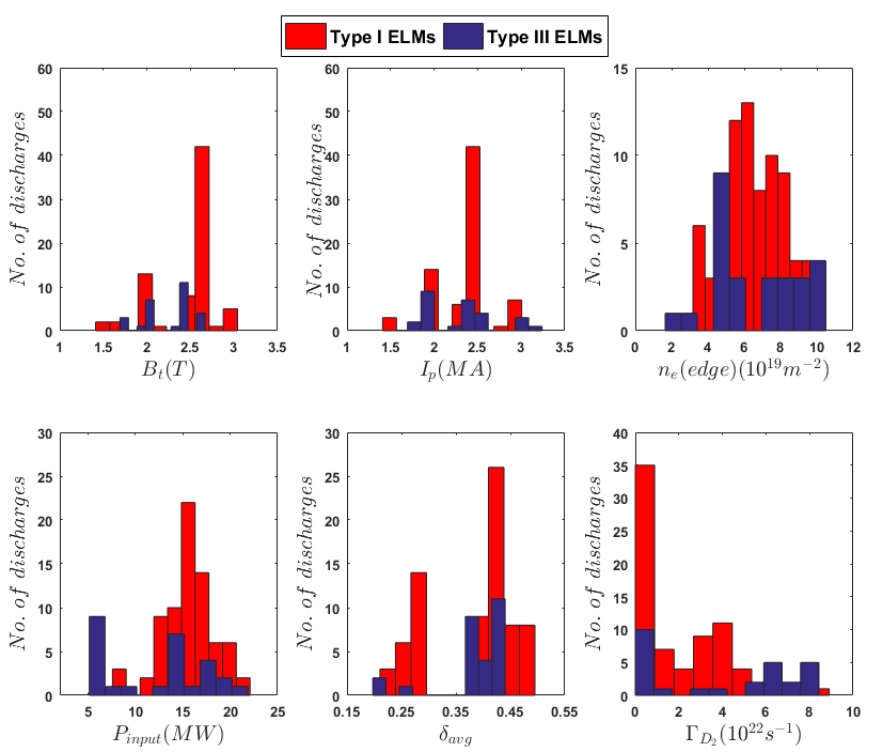

Figure 1: Histograms of plasma parameters in the analyzed dataset.

The global plasma parameters considered herein are: vacuum toroidal field at $R=2.96\left(B_{t}, \mathrm{~T}\right)$, plasma current $\left(I_{p}, \mathrm{MA}\right)$, line integrated edge density $\left(n_{e}, 10^{19} \mathrm{~m}^{-2}\right)$, gas fueling $\left(\Gamma_{D_{2}}, 10^{22} \mathrm{~s}^{-1}\right)$, input power $\left(P_{\text {input }}, \mathrm{MW}\right)$ and avno erage triangularity $\left(\delta_{\text {avg }}\right)$. Histograms of plasma parameters for each class are presented in Figure 1. From a visual inspection of Figure 1, considerable overlap amongst the two classes can be readily observed, indicating a non-trivial classification problem.

Performance assessment. Leave-one-out cross validation is used for assessing generalization capability of the classifier. For a dataset with $N$ plasmas, $N$ iterations are performed where $N-1$ plasmas are used for training and the remaining sample is used for testing. The leave-oneout cross validated (CV) success rate, which here is quoted as the percentage of ELMy plasmas correctly classified, is in effect, an estimation of the expected performance of the classifier on an unknown independent dataset.

Predictive capability of each plasma parameter. LDA is performed on the plasmas represented by each global plasma parameter individually. In this case, the estimated covariance matrices coincide with the variances of the two classes and the discriminant function is reduced to a discriminating value (DV). This DV, derived under the assumption of equal variances, is then applied for classification. The DV is given as:

$$
D V=\frac{1}{2}\left(\mu_{\text {class } 1}+\mu_{\text {class } 2}\right) \text {. }
$$

The leave-one-out CV success rates (\%) and DVs are presented in Table 1. Under the assumption of unequal variances (QDA), the analysis produces success rates similar to those presented in Table 1 (differences are $\sim 1 \%$ ). Further, Table 1 reveals that the parameters $P_{\text {input }}$ and $\Gamma_{D_{2}}$ yield 


\begin{tabular}{ccc}
\hline Plasma & $\begin{array}{c}\text { Leave-one-out CV } \\
\text { success rate } \%)\end{array}$ & DV \\
parameters & 73.0 & 2.35 \\
$B_{t}(\mathrm{~T})$ & 74.0 & 2.34 \\
$I_{p}(\mathrm{MA})$ & 74.0 & 6.45 \\
$n_{e}\left(10^{19} m^{-2}\right)$ & 82.0 & 13.7 \\
$P_{\text {input }}(\mathrm{MW})$ & 81.0 & 2.99 \\
$\Gamma_{D_{2}}\left(10^{22} s^{-1}\right)$ & 74.0 & 0.384 \\
$\delta_{\text {avg }}$ & & \\
\hline
\end{tabular}

Table 1: Predictive capability of individual plasma parameters using Linear discriminant analysis.

\begin{tabular}{ccccc}
\hline \multirow{2}{*}{$\begin{array}{c}\text { Plasma } \\
\text { parameters }\end{array}$} & & \multicolumn{3}{c}{$\begin{array}{c}\text { Leave-one-out CV } \\
\text { success }(\%)\end{array}$} \\
\cline { 3 - 5 }$P_{\text {input }}, \Gamma_{D_{2}}$ & LDA & 94.6 & 80.8 & 91.0 \\
& QDA & 90.5 & 84.6 & 89.0 \\
\hline \multirow{2}{*}{$P_{\text {input }}, \Gamma_{D_{2}}, I_{p}$} & LDA & 94.6 & 76.9 & 90.0 \\
& QDA & 91.9 & 80.8 & 89.0 \\
\hline \multirow{2}{*}{$P_{\text {input }}, \Gamma_{D_{2}}, \delta_{\text {avg }}$} & LDA & 94.6 & 80.8 & 91.0 \\
& QDA & 91.9 & 73.1 & 87.0 \\
\hline \multirow{2}{*}{$P_{\text {input }}, \Gamma_{D_{2}}, n_{e}$} & LDA & 93.2 & 76.9 & 89.0 \\
\hline \multirow{2}{*}{$P_{\text {input }}, \Gamma_{D_{2}}, B_{t}$} & QDA & 90.5 & 80.8 & 88.0 \\
\hline$P_{\text {input }}, \Gamma_{D_{2}}, \delta_{\text {avg }}$, & QDA & 90.5 & 80.8 & 88.0 \\
$B_{t}, I_{p}$ & QDA & 93.2 & 84.6 & 91.0 \\
\hline$P_{\text {input }}, \Gamma_{D_{2}}, n_{e}$, & LDA & 94.6 & 84.6 & 92.0 \\
$B_{t}, I_{p}$ & QDA & 94.6 & 84.6 & 93.0 \\
\hline$P_{\text {input }}, \Gamma_{D_{2}}, \delta_{\text {avg }}$, & LDA & 94.6 & 84.6 & 94.0 \\
$B_{t}, I_{p}, n_{e}$ & QDA & 96.0 & 88.5 & 94.0 \\
\hline
\end{tabular}

Table 2: Average and class-wise leave-one-out CV success (\%) for a linear and quadratic combination of plasma parameters obtained by LDA and QDA, respectively.

the highest success rates and hence may play the main role in the classification between the two classes. This corresponds with established knowledge regarding ELM occurrence.

Combinations of plasma parameters. DA is then performed on the linear and quadratic combinations of the plasma parameters, in order to further improve the suc-

rate. The average and class-wise leave-one-out $\mathrm{CV}$ success rates are given in Table 2 . It can be noted that a linear combination of $P_{\text {input }}$ and $\Gamma_{D_{2}}$ improves the average leave-one-out CV success rate to $91.0 \%$ from $(81.0$ - 82.0$) \%$ yielded by each of them individually. On the other hand,

140 a quadratic combination of $P_{\text {input }}$ and $\Gamma_{D_{2}}$ increases the 170 average success rate to $89.0 \%$. This is further illustrated in Figure 2, It can be readily observed that the vertical and horizontal dashed lines discriminate the two classes poorly, whereas the solid lines, which are a function of $P_{\text {input }}$ and

$\Gamma_{D_{2}}$, better separate the two classes. Further, it can be ${ }_{17}$ seen that for $10.4 \mathrm{MW} \leq P_{\text {input }} \leq 16.5 \mathrm{MW}$ the difference between the quadratic and linear boundary is small

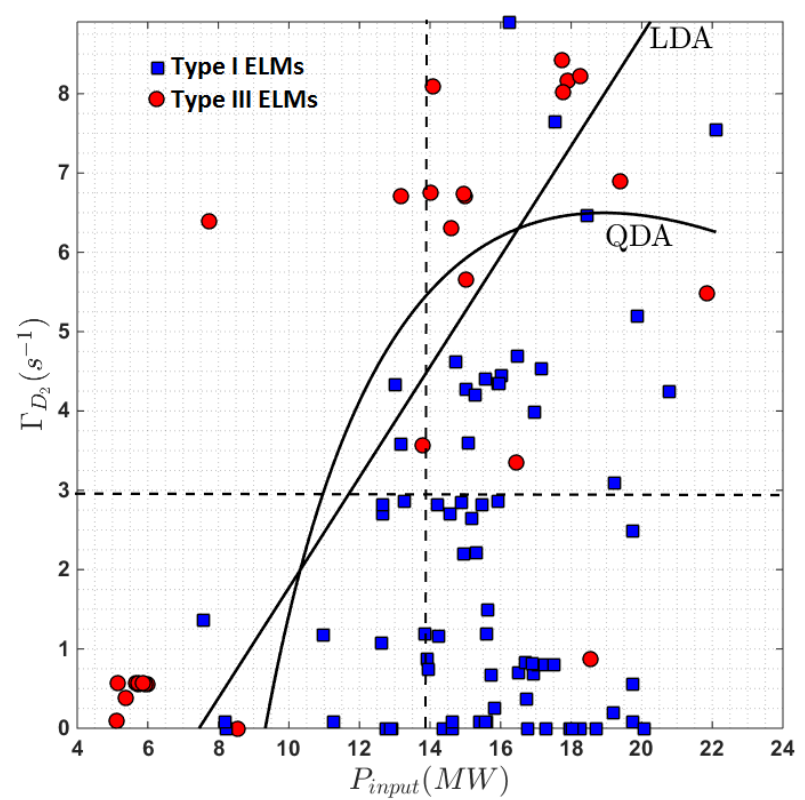

Figure 2: The solid line and curve indicate the linear (LDA) and the quadratic (QDA) discriminant functions for type I and type III ELMs from the analysed plasmas. Vertical and horizontal dashed lines mark the discriminating values for $P_{\text {input }}$ and $\Gamma_{D_{2}}$, respectively.

$\left(\Delta \Gamma_{D_{2}} \leq 1.0 s^{-1}\right)$. However, for $P_{\text {input }}>16.5 M W$, this difference is substantial.

Figure 3, presents the decrease in error rate (\%) with the addition of other plasma parameters. An addition of the remaining 4 plasma parameters, $B_{t}, I_{p}, n_{e}$ and $\delta_{a v g}$ to $P_{\text {input }}$ and $\Gamma_{D_{2}}$ reduces the average error rate to $8 \%$ (alternatively, average success rate improves to $92 \%$ ) for quadratic case. While the addition of $\Gamma_{D_{2}}$ to $P_{\text {input }}$ had reduced the error rate by a factor of $\sim 2$, the addition of the remaining 4 parameters only lowers it further by $1 \%$ for LDA and $5 \%$ for QDA. It is noteworthy that the error rate for type III ELMs reduces by 4\% for both LDA and QDA whereas the error rate for type I ELMs remains unchanged for LDA and lowers by $\sim 5 \%$ for QDA. However, this reduction in error rates comes at the expense of an increased model complexity brought about by an increase in the number of parameters in the discriminant function.

From the various models analyzed, the linear combination of $P_{\text {input }}, \Gamma_{D_{2}}, B_{t}, I_{p}$ along with either $n_{e}$ or $\delta_{a v g}$, can be considered best models as they yield high average and class-wise success rates with the least number of parameters. The quadratic combination of $P_{\text {input }}, \Gamma_{D_{2}}, B_{t}$, $I_{p}$ and $n_{e}$ gives a slightly higher success rate amongst all analyzed models. However, the quadratic model is significantly more complex, less intuitive and less tractable, than the linear counterpart. However, if the primary goal is correct classification of a new plasma, then this quadratic model can be slightly advantageous compared to the linear ones. 

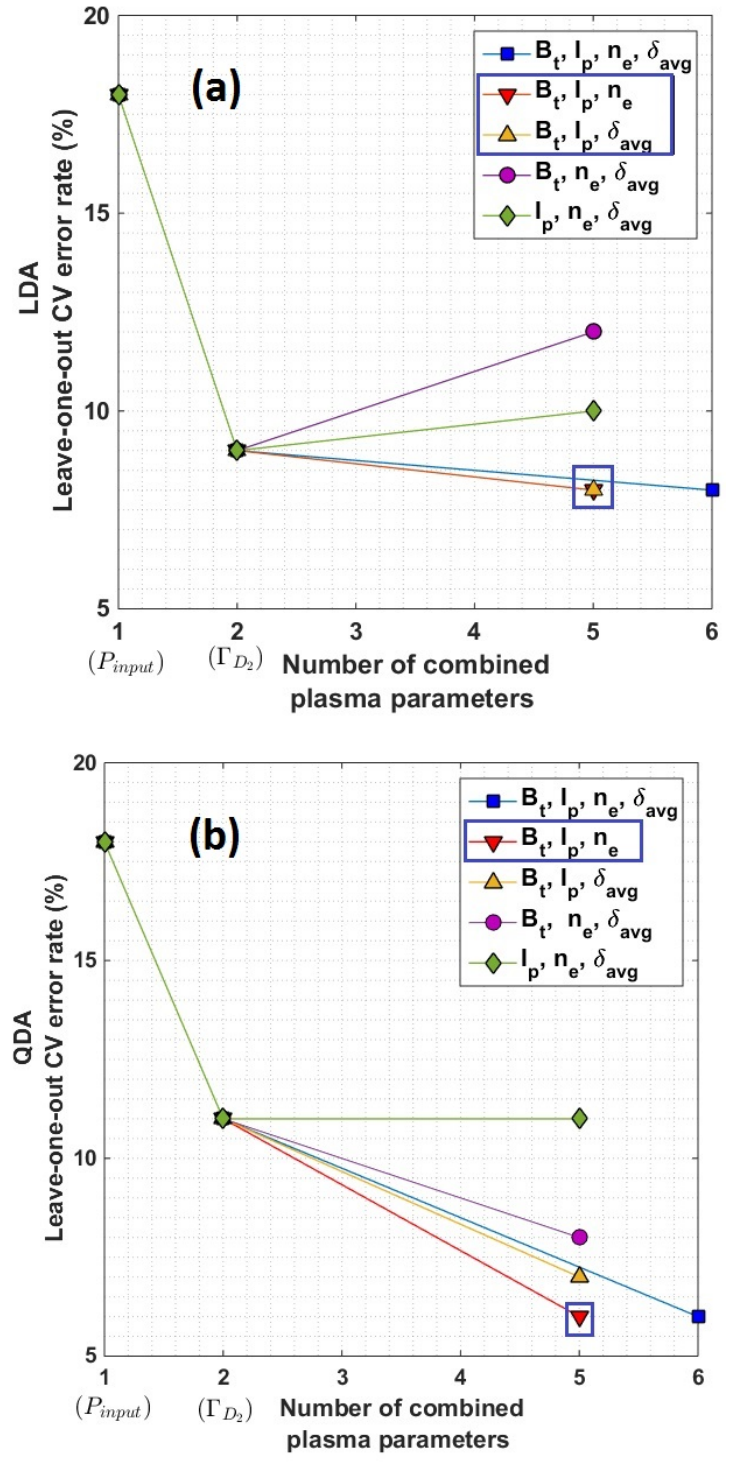

Figure 3: Leave-one-out CV error rate versus the number of com- $^{210}$ bined plasma parameters using (a) LDA, (b) QDA

Separation hyperplane for type I and type III ELMs. The ${ }_{215}$ mathematical form for the linear discriminant functions derived for the classification of type I and III ELMs is presented in Table 3 . The classification success rates for these linear separating hyperplanes (boundary) are provided in in $_{20}$ Table 2. For each of the discriminant functions, given in Table 3, type III ELMs are expected if the left-hand side of expression is less than the constant on the right-hand side. Otherwise, type I ELMs are expected.

\section{Conclusions}

In this work, a simple, high-accuracy, standardized au- ${ }^{230}$ tomated classifier has been presented which can considerably reduce the effort of ELM experts in identifying ELM types. Further, the classifier provides a separation hyperplane in terms of plasma parameters which reflects under- ${ }^{235}$

\begin{tabular}{ccc}
\hline & Linear discriminant functions & Wilks' $\Lambda$ \\
\hline L1 & $P_{\text {input }}-1.41 \Gamma_{D_{2}}=7.47$ & 0.60 \\
\hline L2 & $P_{\text {input }}-1.25 \Gamma_{D_{2}}+7.06 B_{t}$ & 0.53 \\
& $-8.81 I_{p}+0.70 n_{e}=8.75$ \\
\hline L3 & $P_{\text {input }}-0.765 \Gamma_{D_{2}}+12.4 B_{t}$ \\
& $-10.7 I_{p}-26.1 \delta_{\text {avg }}=3.96$ & 0.47 \\
\hline
\end{tabular}

Table 3: Linear separation hyperplanes (boundary) for type I/III ELMs, in terms of global plasma parameters. Type III ELMs are expected if the left-hand side of the expression is less than the constant on the right-hand side. The corresponding classification success rates (\%) are provided in Table 2 Wilks' $\Lambda$ indicates the goodness-of-fit of each discriminant function. A value less than about 0.63 implies a reasonably good fit.

lying physics and can also aid in determining the operational boundaries for ELMy regimes during experimental planning.

The future work will involve an expansion of the dataset as well as the use of normalized global plasma parameters for rendering a machine independent classifier of ELM types.

$[2]$

A. Murari, F. Pisano, J. Vega, B. Cannas, A. Fanni, S. Gonzalez, M. Gelfusa, M. Grosso, Extensive statistical analysis of ELMs on JET with a carbon wall, Plasma Physics and Controlled Fusion 56 (11) (2014) 114007. doi:10.1088/0741-3335/56/11/114007

[3] N. Duro, R. Dormido, J. Vega, S. Dormido-Canto, G. Farias, J. Sanchez, H. Vargas, A. Murari, Automated recognition system for ELM classification in JET, Fusion Engineering and Design

$84(2-6)$ (2009) 712-715. doi:10.1016/j.fusengdes.2008.12. 003 .

[4] A. Shabbir, G. Verdoolaege, J. Vega, A. Murari, ELM Regime Classification by Conformal Prediction on an Information Manifold, IEEE Transactions on Plasma Science (2015) 1-10 doi: 10.1109/TPS.2015.2489689

[5] A. Shabbir, G. Hornung, G. Verdoolaege, JET Contributors, A classification scheme for edge-localized modes based on their probability distributions, Review of Scientific Instruments 87 (11) (2016) 11D404. doi:10.1063/1.4955479

[6] O. J. W. F. Kardaun, Classical Methods of Statistics, SpringerVerlag, 2005. doi:10.1007/3-540-29288-8

[7] A. Shabbir, G. Verdoolaege, O. J. W. F. Kardaun, J.-M. Noterdaeme, Visualization of the operational space of edge-localized modes through low-dimensional embedding of probability distributions, Review of Scientific Instruments 85 (11) (2014) 11E819. doi:10.1063/1.4892866 